\title{
Species-Specific Immunity Induced by Infection with Entamoeba histolytica and Entamoeba moshkovskii in Mice
}

\author{
Chikako Shimokawa ${ }^{1}$, Richard Culleton ${ }^{2}$, Takashi Imai ${ }^{3}$, Kazutomo Suzue ${ }^{3}$, Makoto Hirai ${ }^{3}$, Tomoyo \\ Taniguchi $^{3}$, Seiki Kobayashi ${ }^{4}$, Hajime Hisaeda ${ }^{3}$, Shinjiro Hamano ${ }^{1,5^{*}}$
}

1 Department of Parasitology, Nagasaki University, Nagasaki, Japan, 2 Malaria Unit, Institute of Tropical Medicine (NEKKEN), Nagasaki University, Nagasaki, Japan, 3 Department of Parasitology, Graduate School of Medicine, Gunma University, Gunma, Japan, 4 Department of Tropical Medicine and Parasitology, Keio University School of Medicine, Shinjuku, Tokyo, Japan, 5 Global COE Program, Nagasaki University, Nagasaki, Japan

\begin{abstract}
Entamoeba histolytica, the parasitic amoeba responsible for amoebiasis, causes approximately 100,000 deaths every year. There is currently no vaccine against this parasite. We have previously shown that intracecal inoculation of E. histolytica trophozoites leads to chronic and non-healing cecitis in mice. Entamoeba moshkovskii, a closely related amoeba, also causes diarrhea and other intestinal disorders in this model. Here, we investigated the effect of infection followed by drug-cure of these species on the induction of immunity against homologous or heterologous species challenge. Mice were infected with E. histolytica or E. moshkovskii and treated with metronidazole 14 days later. Re-challenge with $E$. histolytica or $E$. moshkovskii was conducted seven or 28 days following confirmation of the clearance of amoebae, and the degree of protection compared to non-exposed control mice was evaluated. We show that primary infection with these amoebae induces a species-specific immune response which protects against challenge with the homologous, but not a heterologous species. These findings pave the way, therefore, for the identification of novel amoebae antigens that may become the targets of vaccines and provide a useful platform to investigate host protective immunity to Entamoeba infections.
\end{abstract}

Citation: Shimokawa C, Culleton R, Imai T, Suzue K, Hirai M, et al. (2013) Species-Specific Immunity Induced by Infection with Entamoeba histolytica and Entamoeba moshkovskii in Mice. PLoS ONE 8(11): e82025. doi:10.1371/journal.pone.0082025

Editor: Ikuo Igarashi, Obihiro University of Agriculture and Veterinary Medicine, Japan

Received August 26, 2013; Accepted October 24, 2013; Published November 29, 2013

Copyright: ( 2013 Shimokawa et al. This is an open-access article distributed under the terms of the Creative Commons Attribution License, which permits unrestricted use, distribution, and reproduction in any medium, provided the original author and source are credited.

Funding: The work was supported by a Grant-in-Aid for Scientific Research on Priority Areas from MEXT (21022037 to S.H.), http://www.mext.go.jp/ english/, Grants-in-Aid for International Scientific Research (B) from JSPS (20406008, 23406009 to S.H.), http://www.jsps.go.jp/english/index.html, a Health Labour Sciences Research Grant (H20-Shinkoh-Ippan-016, H23-Shinkoh-Ippan-014 to S.H.), http://www.mhlw.go.jp/english/, the Takeda Foundation http:// www.takeda-sci.or.jp/, the Uehara Foundation, (to S. H.) http://www.ueharazaidan.or.jp/, the Global COE Program, Nagasaki University, supported by MEXT (to S. H.) http://www.jsps.go.jp/j-globalcoe/ and the Sasakawa Foundation (Scientific Research Grant from The Japan Science Society) (to C.S.), http://www.jss.or.jp/ikusei/sasakawa/. The funders had no role in study design, data collection and analysis, decision to publish, or preparation of the manuscript.

Competing interests: The authors have declared that no competing interests exist.

*E-mail: shinjiro@nagasaki-u.ac.jp

\section{Introduction}

Amoebiasis, an infectious disease caused by the parasitic protozoan Entamoeba histolytica is responsible for over 50 million cases in tropical regions and nearly 100,000 deaths worldwide each year. Infection is initiated through the ingestion of cysts in contaminated food or water. E. histolytica primarily infects the intestine, and may cause a wide range of symptoms from mild diarrhea to serious dysentery. If untreated, the parasite can cause life-threatening hemorrhagic colitis and/or extra-intestinal abscesses [1-5].

E. histolytica trophozoites are able to colonize the human intestine by adhering to colonic mucins and subsequently to epithelial cells via cell surface lectin [6]. This lectin is important for colonic colonization by $E$. histolytica. A colonizationblocking vaccine targeting this parasite lectin could prevent trophozoite adherence and thus provide protection against subsequent invasive disease [7]. Furthermore, recently, it has also been reported that there is a correlation between the presence of anti-lectin fecal immunoglobulin A ( $\lg A)$ antibodies and protection from parasitic colonization in humans and mice [7-9]. These reports suggest that amoebiasis can be controlled by acquired immunity.

Entamoeba moshkovskii is closely related to Entamoeba dispar and E. histolytica and is microscopically indistinguishable from them in its cyst and trophozoite forms [10]. Recently, we reported that E. moshkovskii causes diarrhea, colitis and weight loss in mice, and that in 
Bangladeshi children, acquisition of E. moshkovskii infection was associated with diarrhea [11].

Here, using E. histolytica and E. moshkovskii infections in mice, we evaluate whether the immunity against reinfection that occurs following a primary infection is species-specific. We find that, following a primary infection with either $E$. histolytica or E. moshkovskii, mice are protected from re-challenge with a homologous species, but remained susceptible to a heterologous species. These results show, for the first time, that the immunity acquired during primary infection with Entamoeba spp. confers species-specific protective immunity.

\section{Materials and Methods}

\section{Mice}

Male CBA/J mice were purchased from Jackson Laboratories. Animals were maintained under specific pathogen free conditions at the Animal Research Center for Tropical Infectious Diseases, Nagasaki University, and were challenged when they were 5-8 weeks old. All experiments that involved mice were reviewed and approved by the Committee for Ethics on Animal Experiments of the Graduate School of Nagasaki University, and were conducted under the control of the Guidelines for Animal Experiments in the Graduate School of Medicine, Nagasaki University, and the Law (No. 105) and Notification (No. 6) of the Japanese Government pertaining to the use of experimental animals.

\section{Parasite Culture and Infection}

Trophozoites of E. histolytica, originally laboratory strain HM1:IMSS (American Type Culture Collection, Manassas, VA), were from Prof. Eric Houpt, University of Virginia, and were serially passaged in vivo through the ceca of mice [12]. Trophozoites of the E. moshkovskii Laredo strain, were a gift from Dr. Seiki Kobayashi, Keio University, School of Medicine (originally from the late professor Louis S. Diamond, $\mathrm{NIH}$, Bethesda, Maryland). Cecal contents were cultured at $37^{\circ} \mathrm{C}$ and $25^{\circ} \mathrm{C}$, respectively, in BIS-33 medium supplemented with heat-inactivated $10 \%$ adult bovine serum, $25 \mathrm{U} / \mathrm{ml}$ penicillin and $25 \mathrm{mg} / \mathrm{ml}$ streptomycin [13]. Trophozoites in the logarithmic growth phase were used in the experiments.

\section{Intracecal inoculation of Entamoeba spp}

Trophozoites of E. histolytica HM1:IMSS and E. moshkovskii Laredo strain were collected after incubating the tubes on ice for 5-10 minutes. Then, the number of trophozoites was counted. We anesthetized mice with Domitor (medetomidine hydrochloride: $0.1 \mathrm{mg} / \mathrm{kg}$ ) and Dormicum (midazolam: $0.1 \mathrm{mg} /$ $\mathrm{kg}$ ), shaved their abdomens to incise the skin, exteriorized each cecum from the peritoneum, and injected $150 \mu$ l of $1 \times 10^{6}$ trophozoites into the apical sites of cecum. Then, the cecum was blotted and the peritoneum and the skin were sutured. Mice were kept on warming blankets at $37^{\circ} \mathrm{C}$ throughout surgery. Survival rates were $\geq 90 \%$ in all mice.

\section{Detection of each Entamoeba spp. by PCR using DNA extracted from stool of mice}

For isolation of Entamoeba DNA from mouse stools, QIAamp DNA Stool Kits (QIAGEN, Valencia CA) were used according to manufacturer's instructions. The primer sequences used for PCR are as previously described [14].

\section{Administration of metronidazole}

For in vivo studies, stock solutions of metronidazole (Sigma Aldrich, St. Louis, MO) were prepared in $100 \%$ dimethyl sulfoxide at a concentration of $10 \mathrm{mg} / \mathrm{mL}$ and stored at $4^{\circ} \mathrm{C}$. The stock solution was diluted 32 times with distilled water to $0.3125 \mathrm{mg} / \mathrm{mL}$, in which the concentration of DMSO was $3.125 \%$. Mice were treated orally with metronidazole at a dose of $12.5 \mathrm{mg} / \mathrm{kg}$ of body weight. To cure primary infections with $E$. histolytica or with $E$. moshkovskii, all of mice challenged with $E$. histolytica or with $E$. moshkovskii were treated with $1 \mathrm{~mL}$ of metronidazole orally $(0.3125 \mathrm{mg} / \mathrm{mL})$ using gastric intubation on day 14 post-infection. Naïve mice were also administered with metronidazole and used as control.

\section{Statistical analysis}

Differences between groups were analyzed for statistical significance with unpaired Student's $t$-test and $X^{2}$ test. All of these were performed using Excel software. Probabilities below 0.05 were considered statistically significant.

\section{Results}

\section{E. moshkovskii infections were resolved earlier than $E$. histolytica infections}

We have previously demonstrated that $\mathrm{C} 3 \mathrm{H} / \mathrm{HeN}, \mathrm{C} 3 \mathrm{H} / \mathrm{HeJ}$ and CBA/J mice allow the establishment of $E$. histolytica and $E$. moshkovskii infections, while many strains of mice including C57BL/6 and BALB/C mice do not, indicating that susceptibility to $E$. histolytica and $E$. moshkovskii infection is dependent on the genetic background of the host [11,12,15-17]. Trophozoites of E. histolytica and E. moshkovskii were intracecally inoculated into $\mathrm{CBA} / \mathrm{J}$ mice. As expected, both $E$. histolytica and $E$. moshkovskii succeeded in infecting CBA/J mice after challenge (Figure 1). E. histolytica infected the ceca in approximately $80 \%$ of CBA/J mice (16 of 20 ) as confirmed by both culture and PCR of intracecal contents two days after challenge. In contrast, E. moshkovskii infected the ceca of CBA $/ J$ mice in approximately $65 \%$ of mice (13 of 20 ) at the same point. At day 14 post-challenge, the infection rate of $E$. histolytica was approximately $60 \%$ (12 of 20 mice positive), though that of E. moshkovskii was approximately $5 \%$ of mice (1 of 20). At 21 days post-challenge, the infection rate of $E$. histolytica was approximately $58 \%$ (11 of 19 ) and that of $E$. moshkovskii was $0 \%$.

\section{Metronidazole Sensitivity in E. moshkovskii}

So as to treat mice infected with $E$. histolytica and $E$. moshkovskii, the effect of metronidazole on the growth and survival of E. histolytica and E. moshkovskii trophozoites was evaluated in vitro. The number of viable cells in glass tubes 
Figure. 1

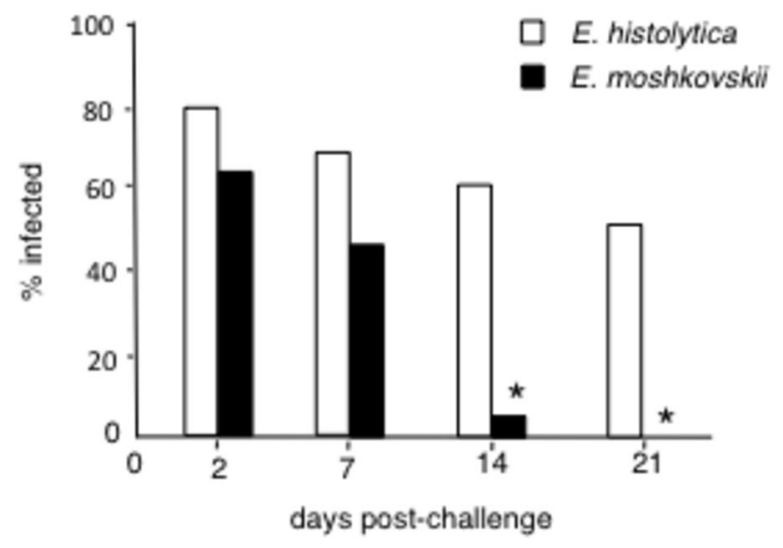

Figure 1. Entamoeba moshkovskii infections were resolved earlier than Entamoeba histolytica infections. CBA/J mice were intracaecally infected with $1 \times 10^{6}$ trophozoites of $E$. histolytica and E. moshkovskii. Infection rate was monitored by detecting amoebae in caecal content and by amplifying the amoeba gene from faecal DNA on days $2,7,14$, and 21 . Infection rate of mice with $E$. histolytica and E. moshkovskii was shown as open and closed columns, respectively. Values show the representative result out of 3 individual experiments. Asterisks indicate statistical significance with $p<0.05$ between mice infected with $E$. histolytica and E. moshkovskii by $\mathrm{x}^{2}$ test.

doi: 10.1371/journal.pone.0082025.g001

\section{Figure 2}

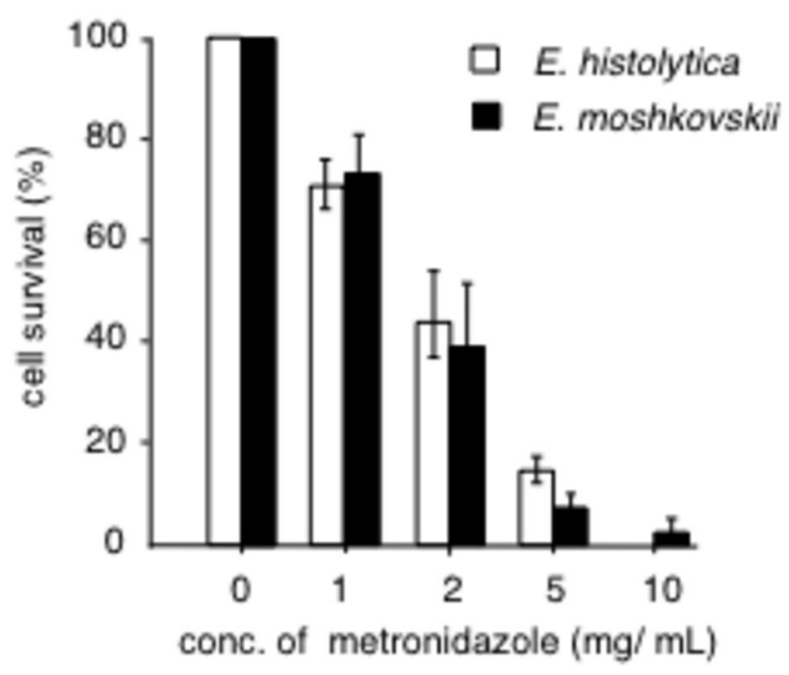

Figure 2. The effect of metronidazole on the growth and survival of Entamoeba histolytica and Entamoeba moshkovskii trophozoites was evaluated in vitro. E. histolytica and E. moshkovskii were incubated with various concentrations of metronidazole for $48 \mathrm{~h}$. Then, the number of viable cells was counted and the proportion of it versus initial number was shown as open and closed columns, respectively.

doi: 10.1371/journal.pone.0082025.g002

was counted after incubation of $E$. histolytica and $E$. moshkovskii with various concentrations of metronidazole for 48h. The numbers of E. histolytica and E. moshkovskii treated with metronidazole decreased significantly in a dose dependent manner (Figure 2).

\section{Amoebic infection induced species-specific protective immunity}

In order to examine whether protection against re-infection can be induced by primary infection, the mice that allowed the establishment of the primary infection with $E$. histolytica or $E$. 
Figure 3

A

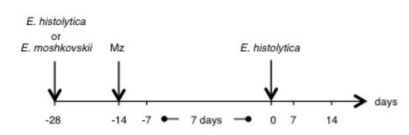

C

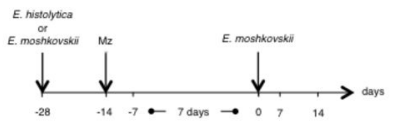

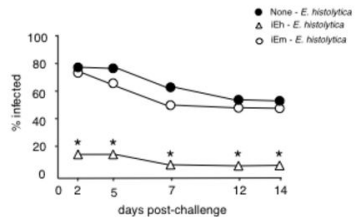

D

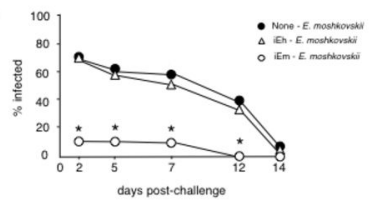

Figure 3. Amoebic infection induced species-specific protective immunity. Mice were infected with $1 \times 10^{6}$ trophozoites of Entamoeba histolytica (open triangle) or Entamoeba moshkovskii (open circle) and treated with metronidazole (Mz) on day 14 following induction of the primary infection. The clearance of amoeba was confirmed seven days after treatment by PCR. Mice were kept without any intervention for an additional week, and then re-challenged with $1 \times 10^{6}$ trophozoites of $E$. histolytica (A, B) or $E$. moshkovskii $(\mathrm{C}, \mathrm{D})$ at 14 days after treatment. The number of mice used was as follows: for naïve $\rightarrow E$. histolytica, $\mathrm{N}=16:$ for $E$. histolytica $\rightarrow E$. histolytica, $\mathrm{N}=20$ : for $E$. moshkovskii $\rightarrow E$. histolytica, $\mathrm{N}=20$ (A, B); for naïve $\rightarrow E$. moshkovskii, $\mathrm{N}=20$ : for $E$. histolytica $\rightarrow E$. moshkovskii, $\mathrm{N}=20$ : for $E$. moshkovskii $\rightarrow E$. moshkovskii, $\mathrm{N}=20$ (C, D). Asterisks indicate statistical significance with $\mathrm{p}<0.05$ by $\mathrm{X}^{2}$ test between mice infected with $E$. histolytica and $E$. moshkovskii in the primary infection.

doi: 10.1371/journal.pone.0082025.g003

moshkovskii were treated with metronidazole on day 14 and used for the secondary challenge. The clearance of amoeba was confirmed seven days after treatment by PCR. Mice were kept without any intervention for an additional week, and then re-challenged with $E$. histolytica or E. moshkovskii a total of 14 days after treatment (Figure $3 \mathrm{~A}, \mathrm{C}$ ). The mice infected with $E$. histolytica and treated with metronidazole showed resistance to homologous re-challenge infection (Figure 3B), but allowed establishment of infection with the heterologous species $E$. moshkovskii in a manner similar to that seen in naive mice (Figure 3D). Similarly, mice infected with E. moshkovskii and treated with metronidazole showed resistance to homologous re-challenge infection with E. moshkovskii (Figure 3D), but allowed the establishment of infection with the heterologous species E. histolytica (Figure 3B). Thus, mice that experienced primary amoebic infection acquired resistance to secondary homologous species infection. However, primary amoebic infection did not confer protection against heterologous species secondary infection. These results show that intestinal amoebic infection induces species-specific protective immunity.

\section{The protection induced by primary infection lasts more than four weeks}

To examine how long the protection observed against secondary infection lasts, mice were re-challenged with homologous or heterologous amoebae on day 35 after treatment with metronidazole 14 days after primary infection. The clearance of amoeba was confirmed seven days after treatment by PCR (Figure 4A, C). As shown in Figure 4B and $4 D, 35$ days after the treatment of the primary infection, mice were resistant to homologous re-challenge, but were susceptible to heterologous species infection. Mice kept for 35 days after the treatment of the primary infection showed increased infection rates compared to those kept just for 14 days, a phenomenon that was most apparent on day 2 post rechallenge in the case of $E$. histolytica and on days 2 and 5 post challenge with E. moshkovskii (Figure 4B and 4D). These results suggest that the protection induced by primary infection may include not only memory responses but also remaining primary immune responses, both of which are species specific.

\section{Infection-induced species-specific immunity protects mice from weight loss}

During the primary infection, mice infected with $E$. moshkovskii suffered severe symptoms. Following re-challenge with E. moshkovskii (homologous species) 14 days after treatment of the primary infection, mice did not show any weight loss (Figure 5A). Slight weight loss was observed, however, in mice re-challenged 35 days after treatment, but the severity of weight loss was much smaller than that observed during the primary infection (Figure 5B). The weight loss was also ameliorated in mice re-infected with $E$. histolytica, when having been given a primary infection with the homologous species (data not shown).

\section{Discussion}

Our results clearly demonstrate that exposure to, and subsequent drug clearance of, the parasitic amoebae $E$. histolytica and E. moshkovskii invokes a strong immune response that protects mice from subsequent infection with a homologous species. This protection is species specific, affording little to no protection against a heterologous species challenge. The fact that this strong species-specific immunity was shown to last at least 35 days following the treatment of the initial infection suggests that memory responses are involved. 


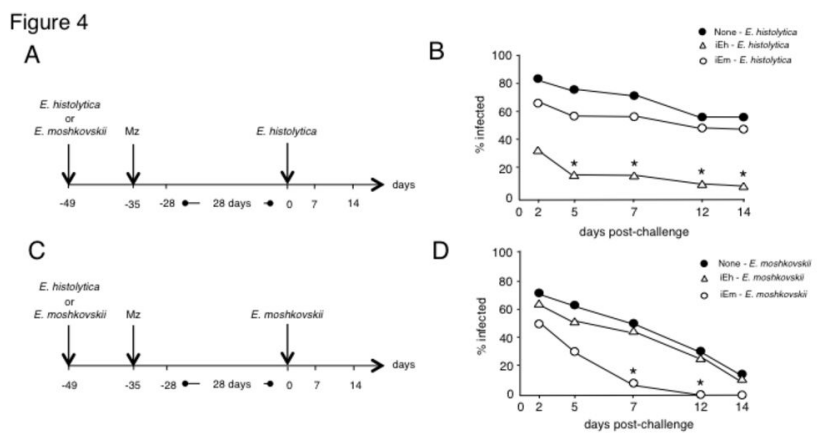

Figure 4. The protection induced by primary infection lasts more than four weeks. Mice were infected with $1 \times 10^{6}$ trophozoites of Entamoeba histolytica (open triangle) or Entamoeba moshkovskii (open circle) and treated with metronidazole (Mz) on day 14 following induction of the primary infection. The clearance of amoeba was confirmed seven days after treatment by PCR. Mice were kept without any intervention for 28 days, and re-challenged with $1 \times 10^{6}$ trophozoites of $E$. histolytica (A, B) or $E$. moshkovskii $(\mathrm{C}, \mathrm{D})$ at 35 days after treatment. The number of mice used was as follows: for naïve $\rightarrow E$. histolytica, $\mathrm{N}=16$ : for $E$. histolytica $\rightarrow E$. histolytica, $\mathrm{N}=20$ : for $E$. moshkovskii $\rightarrow E$. histolytica, $\mathrm{N}=26(\mathrm{~A}, \mathrm{~B})$; for naïve $\rightarrow E$. moshkovskii, $\mathrm{N}=15$ : for $E$. histolytica $\rightarrow E$. moshkovskii, $\mathrm{N}=20$ : for $E$. moshkovskii $\rightarrow$ E. moshkovskii, $\mathrm{N}=20$ (C, D). Asterisks indicate statistical significance with $\mathrm{p}<0.05$ by $\mathrm{X}^{2}$ test between mice infected with $E$. histolytica and $E$. moshkovskii in the primary infection.

doi: 10.1371/journal.pone.0082025.g004

Figure 5
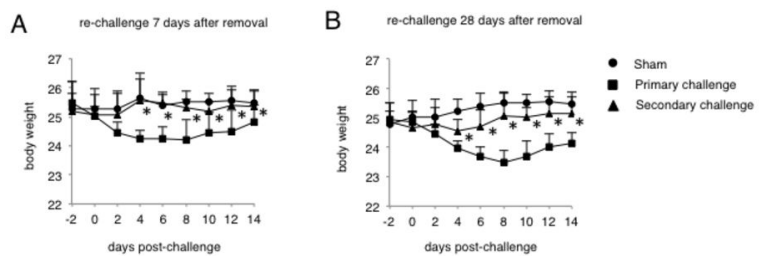

Figure 5. The change of body weight was monitored after re-challenging mice with Entamoeba moshkovskii. The naïve mice or mice exposed to primary E. moshkovskii infection were re-challenged with $1 \times 10^{6}$ trophozoites of E. moshkovskii at 7 or 28 days after confirming the clearance of primary infection. The studies were repeated 3 times with similar results. Asterisks indicate statistical significance with $p<0.05$ between the groups of mice with primary and secondary infection using unpaired Student's $t$ test.

doi: 10.1371/journal.pone.0082025.g005

Despite the fact that it has long been suspected that people may acquire immunity against amoebae, as older children in endemic areas are infected less frequently than younger children [18], this work constitutes the first experimental proof of this phenomenon. The molecular and cellular mechanisms responsible for the observed protection have not been addressed in this work. Mucosal IgA has been reported to be associated with protection against intestinal amoebiasis in humans, mice and baboons [7,8,19]. Indeed, monoclonal IgA specific for the $E$. histolytica galactose inhibitable adherence (GalNAc) lectin heavy subunit $(\mathrm{HgL})$ is thought to inhibit its interaction with a host sugar moiety in colonic mucins, resulting in the failure of amoebae to settle within the intestines [20]. Furthermore, we and Guo et al. recently reported that IFN- $\gamma$ derived from amoeba-specific $\mathrm{T}$ cells plays a protective role against $E$. moshkovskii (unpublished data) and E. histolytica [21], respectively, suggesting that $T$ cells as well as antibodies specific for amoebic antigens are involved in acquired resistance to intestinal amoebic infections.

The phenomenon of species-specific immunity against parasitic pathogens has been studied in a number of parasitic species, perhaps most comprehensively with the Plasmodium species responsible for malaria [22]. For this pathogen, which exhibits both species and strain specific immunity, antigenic variation of major parasite surface antigens such as the merozoite surface protein 1 (MSP1), induces antibodymediated immune responses that are effective only against the inducing-strain $[22,23]$. Such highly polymorphic strain- and species-specific antigens are thought to evolve through the actions of positive diversifying selection, so that proteins that are targeted by the host immune response rapidly accumulate polymorphisms. Here we show, for the first time, that the phenomenon of species-specific immunity also exists for Entamoeba spp. It seems probable that this is due to 
polymorphisms in major antigen target proteins between species. If so, then such antigens may be identified by comparative genomics. Of particular interest are the GalNAclectin $\mathrm{HgL}$ proteins previously implicated in antibody-mediated protection against $E$. histolytica. Nucleotide sequence comparisons of the genes encoding this protein in $E$ histolytica and E. moshkovskii may shed further light on this.

We found that E. moshkovskii is susceptible to the antiamoeba drug metronidazole both in vitro and in vivo to the same degree as E. histolytica. This finding supports the use of this drug in the treatment of pathogenic E. moshkovskii, and may ease concern of treatment failure following cases of misdiagnosis of E. moshkovskii as E. histolytica.

In summary, we show that exposure to a single drug cured amoebic infection confers resistance to re-challenge with the homologous, but not a heterologous species, for the first time, in which species-specific acquired immunity has been demonstrated for amoebic infections. This work paves the way, therefore, for the identification of novel amoebae antigens that may become the targets of vaccines.

\section{References}

1. Bryce J, Boschi-Pinto C, Shibuya K, Black RE (2005) WHO estimates of the causes of death in children. Lancet 365: 1147-1152. doi:10.1016/ S0140-6736(05)71877-8. PubMed: 15794969.

2. Petri WA Jr., Miller M, Binder HJ, Levine MM, Dillingham R et al. (2008) Enteric infections, diarrhea, and their impact on function and development. J Clin Invest 118: 1277-1290. doi:10.1172/JCI34005. PubMed: 18382740.

3. Guerrant RL, Oriá RB, Moore SR, Oriá MO, Lima AA (2008) Malnutrition as an enteric infectious disease with long-term effects on child development. Nutr Rev 66: 487-505. doi:10.1111/j. 1753-4887.2008.00082.x. PubMed: 18752473.

4. Irusen EM, Jackson TF, Simjee AE (1992) Asymptomatic intestinal colonization by pathogenic Entamoeba histolytica in amebic liver abscess: prevalence, response to therapy, and pathogenic potential. Clin Infect Dis 14: 889-893. doi:10.1093/clinids/14.4.889. PubMed: 1576284.

5. Blessmann J, Ali IK, Nu PA, Dinh BT, Viet TQ et al. (2003) Longitudinal study of intestinal Entamoeba histolytica infections in asymptomatic adult carriers. J Clin Microbiol 41: 4745-4750. doi:10.1128/JCM. 41.10.4745-4750.2003. PubMed: 14532214

6. Chadee K, Petri WA Jr., Innes DJ, Ravdin JI (1987) Rat and human colonic mucins bind to and inhibit adherence lectin of Entamoeba histolytica. J Clin Invest 80: 1245-1254. doi:10.1172/JCI113199. PubMed: 2890655.

7. Houpt E, Barroso L, Lockhart L, Wright R, Cramer C et al. (2004) Prevention of intestinal amebiasis by vaccination with the Entamoeba histolytica Gal/GalNac lectin. Vaccine 22: 611-617. doi:10.1016/ j.vaccine.2003.09.003. PubMed: 14741152.

8. Haque R, Ali IM, Sack RB, Farr BM, Ramakrishnan G et al. (2001) Amebiasis and mucosal IgA antibody against the Entamoeba histolytica adherence lectin in Bangladeshi children. J Infect Dis 183: 1787-1793. doi:10.1086/320740. PubMed: 11372032.

9. Haque R, Duggal P, Ali IM, Hossain MB, Mondal D et al. (2002) Innate and acquired resistance to amebiasis in bangladeshi children. J Infect Dis 186: 547-552. doi:10.1086/341566. PubMed: 12195383.

10. Fotedar R, Stark D, Marriott D, Ellis J, Harkness J (2008) Entamoeba moshkovskii infections in Sydney, Australia. Eur J Clin Microbiol Infect Dis 27: 133-137. doi:10.1007/s10096-007-0399-9. PubMed: 17957394.

11. Shimokawa $C$, Kabir M, Taniuchi M, Mondal D, Kobayashi $S$ et al. (2012) Entamoeba moshkovskii is associated with diarrhea in infants and causes diarrhea and colitis in mice. J Infect Dis 206: 744-751. doi: 10.1093/infdis/jis414. PubMed: 22723640

12. Hamano S, Asgharpour A, Stroup SE, Wynn TA, Leiter EH et al. (2006) Resistance of C57BL/6 mice to amoebiasis is mediated by nonhemopoietic cells but requires hemopoietic IL-10 production. J Immunol 177: 1208-1213. PubMed: 16818779.

13. Diamond LS, Harlow DR, Cunnick CC (1978) A new medium for the axenic cultivation of Entamoeba histolytica and other Entamoeba.

\section{Acknowledgements}

We thank Fumie Hara, Masako Hayashida and Megumi Hamasaki for animal husbandry; Tomoko Takaya, Hiromi Oda and Rumiko Kosugi for administrative support; Richard Culleton for scientific editing; members of Institute of Tropical Medicine, Nagasaki University and Department of Parasitology, Graduate School of Medicine, Gunma University for technical assistance and precious comments.

\section{Author Contributions}

Conceived and designed the experiments: CS SH. Performed the experiments: CS TI KS MH TT SK. Analyzed the data: CS $\mathrm{RC} \mathrm{HH} \mathrm{SH}$. Contributed reagents/materials/analysis tools: CS SK SH. Wrote the manuscript: CS RC SH.

Trans R Soc Trop Med Hyg 72: 431-432. doi: 10.1016/0035-9203(78)90144-X. PubMed: 212851.

14. Hamzah Z, Petmitr S, Mungthin M, Leelayoova S, ChavalitshewinkoonPetmitr P (2006) Differential detection of Entamoeba histolytica, Entamoeba dispar, and Entamoeba moshkovskii by a single-round PCR assay. J Clin Microbiol 44: 3196-3200. doi:10.1128/JCM. 00778-06. PubMed: 16954247.

15. Houpt ER, Glembocki DJ, Obrig TG, Moskaluk CA, Lockhart LA et al. (2002) The mouse model of amebic colitis reveals mouse strain susceptibility to infection and exacerbation of disease by $\mathrm{CD}^{+} \mathrm{T}$ cells. J Immunol 169: 4496-4503. PubMed: 12370386.

16. Asgharpour A, Gilchrist C, Baba D, Hamano S, Houpt E (2005) Resistance to intestinal Entamoeba histolytica infection is conferred by innate immunity and $\mathrm{Gr}-1^{+}$cells. Infect Immun 73: 4522-4529. doi: 10.1128/IAI.73.8.4522-4529.2005. PubMed: 16040963.

17. Hamano S, Becker S, Asgharpour A, Ocasio YP, Stroup SE et al. (2008) Gender and genetic control of resistance to intestinal amebiasis in inbred mice. Genes Immun 9: 452-461. doi:10.1038/gene.2008.37. PubMed: 18480826.

18. Haque R, Mondal D, Duggal P, Kabir M, Roy S et al. (2006) Entamoeba histolytica infection in children and protection from subsequent amebiasis. Infect Immun 74: 904-909. doi:10.1128/IAI. 74.2.904-909.2006. PubMed: 16428733

19. Abd Alla MD, Wolf R, White GL, Kosanke SD, Cary D et al. (2012) Efficacy of a Gal-lectin subunit vaccine against experimental Entamoeba histolytica infection and colitis in baboons (Papio sp.). Vaccine 30: 3068-3075. doi:10.1016/j.vaccine.2012.02.066. PubMed: 22406457.

20. Chadee K, Johnson ML, Orozco E, Petri WA Jr., Ravdin JI (1988) Binding and internalization of rat colonic mucins by the galactose/Nacetyl-D-galactosamine adherence lectin of Entamoeba histolytica. J Infect Dis 158: 398-406. doi:10.1093/infdis/158.2.398. PubMed: 2900266 .

21. Guo X, Stroup SE, Houpt ER (2008) Persistence of Entamoeba histolytica infection in CBA mice owes to intestinal IL-4 production and inhibition of protective IFN-y. Mucosal Immunol 1: 139-146. doi: 10.1038/mi.2007.18. PubMed: 19079171.

22. Inoue M, Tang J, Miyakoda M, Kaneko O, Yui K et al. (2012) The species specificity of immunity generated by live whole organism immunisation with erythrocytic and pre-erythrocytic stages of rodent malaria parasites and implications for vaccine development. Int $\mathrm{J}$ Parasitol 42: 859-870. doi:10.1016/j.ijpara.2012.07.001. PubMed: 22846785.

23. Martinelli A, Cheesman S, Hunt P, Culleton R, Raza A et al. (2005) A genetic approach to the de novo identification of targets of strainspecific immunity in malaria parasites. Proc Natl Acad Sci U S A 102: 814-819. doi:10.1073/pnas.0405097102. PubMed: 15640359. 